\title{
Google, acceso abierto y tecnologías de información y comunicación (TIC) en la economía política contemporánea
}

\author{
Andrew Roth Seneff* \\ EL COLEGIO DE MICHOACÁN
}

El debate entre el modelo económico orientado a la oferta y el modelo orientado a la demanda tiene una relevancia especial para la determinación de los beneficios hechos posibles por las nuevas tecnologías de información y comunicaciones (TIC). En la transición hacia el dominio de modelos orientados a la oferta existen tendencias contenciosas entre iniciativas de corporaciones privadas para la canalización del acceso al conocimiento científico y humanístico, por un lado, y los proyectos que emergen de instituciones del sector público. En varios escenarios actuales se vislumbra este conflicto en relación con la divulgación del conocimiento ahora transformada con las TIC.

(Modelos ecónomicos, Google Book Search, acceso abierto)

\section{INTRODUCCIÓN: OFERTA Y DEMANDA}

$\mathrm{E}$ ntre las diferentes posturas y convicciones que nos separan como ciudadanos en todo el mundo, una actual pero no nueva consiste en la división entre los que apoyan un modelo económico que se basa en la oferta y sus condiciones y otro que se basa en la demanda y sus circunstancias. Con las administraciones de Reagan y Thatcher (1980) que justificaron el arranque de un

* aroth@colmich.edu.mx Agradezco los comentarios críticos de Thomas Calvo a dos versiones de este ensayo. Aun cuando sólo lo dejo abierto como posibilidad por implicación, reconozco un punto valioso de Thomas de que más allá del horizonte de la economía política contemporánea, la creación e institución de bibliotecas digitales nacionales probablemente dependen más de la "excepción cultural" que de "la ley implacable del mercado". No obstante, es importante conocer el contexto político económico de esta excepción. 
proceso de desregulación de las economías nacionales a partir de los problemas económicos de los setenta en el siglo pasado, la postura dominante asociada con las llamadas reformas neoliberales ha estado orientada a la oferta y sus condiciones: a los financiadores de los productores de bienes y especialmente de servicios se les privilegia como el bastión de una economía dedicada a la creación de riqueza. Esta postura se opone a un modelo económico que se basa en la demanda y sus exigencias, asociado con las políticas económicas predicadas por John Maynard Keynes después de la crisis económica de 1929 y en relación con los cambios sociales y económicos introducidos con la segunda guerra mundial. El keynesianismo describe un proceso consumado en políticas sociales dirigidas a la creación de una robusta clase social media y ciertas garantías de bienestar. En una economía orientada a la demanda, el gobierno tiene un papel central, tanto en la regulación de los mercados, como en, por ejemplo, sus programas de seguro social, educación pública gratuita, o programas de gasto para obras públicas e infraestructura nacionales que aseguran una tasa baja de desempleo.

Podemos contemplar el conflicto entre las dos posturas en varios escenarios contenciosos actuales y la intención de este ensayo es describirlo en relación con la oferta y la demanda para la divulgación del conocimiento en las ciencias y humanidades ya transformada con las nuevas tecnologías de información y comunicaciones. Pero antes, es necesario aclarar la relación histórica de crisis y conflicto entre economías de oferta o de demanda y reconocer que el predominio de una o de otra tienen implicaciones muy diferentes para la institucionalización de las ciencias y humanidades en este nuevo siglo. Entonces necesitamos buscar, primero, un caso o escenario en la economía contemporánea que ponga en relieve su contraste con el modelo económico dominante desde la posguerra y hasta el primer lustro de los años setenta del siglo pasado. Así, podemos vislumbrar el dominio actual del capital financiero en el control y canalización de la oferta. Tal predominio se plasma no solamente en su control sobre productos y servicios básicos, sino en la creación de nuevos mercados interconectados y supuestamente equilibrados con recíproca correspondencia a partir de las derivaciones en cómo 
se financia o aseguran el financiamiento de la producción, venta y reventa de tales bienes y servicios.

\section{UNA MIRADA A LA NUEVA ECONOMÍA POLÍTICA DOMINANTE}

La crisis económica que irrumpió en otoño de 2008 fue la más grande y profunda desde la gran depresión económica de 1929. Existen paralelos entre ambas crisis: las dos resultaron después de un crecimiento rápido basado en una oferta no regulada de crédito fácil; en ambas emergió una crisis de confianza en las instituciones de crédito e inversión acompańada por una reducción radical en el consumo de productos y servicios; $y$, también para las dos, el gobierno era la única instancia capaz de estimular la economía mediante una política de gasto para generar el consumo de productos y servicios y reestablecer la confianza en las instituciones económicas. No obstante estos procesos paralelos generales, las condiciones que condujeron a las crisis fueron notablemente diferentes y, también, lo fueron las respuestas a las secuelas a la crisis.

En 1929, una depresión económica grande resultó de un proceso de especulación en la bolsa de valores alimentado por crédito bancario no regulado. No se trataba de desregulación, puesto que los bancos todavía no estaban reglamentados y la creación de un Banco Nacional (o la Reserva Federal en los Estados Unidos) resultó de las reacciones después de la crisis. Existían, además, las doctrinas del voluntarismo y asociacionismo predicadas entre 1924 y 1928 por Herbert Hoover como secretario de Comercio y, luego, de 1929 a 1933 como presidente de la Unión Americana. Los bancos extendieron crédito más allá de su solvencia y de manera semejante inversionistas-deudores manejaron créditos sin la posibilidad productiva de cubrirlos. La recuperación después de 1929 fue orientada explícitamente por un modelo económico keynesiano que introdujo programas de políticas públicas para obras de infraestructura, educación y seguro social.

En 2008, la crisis económica fue también el resultado de especulaciones, pero instrumentadas de una manera muy diferente a la de 1929, tanto en términos tecnológicos, como en términos económi- 
cos. La existencia de las tecnologías de información y comunicaciones permitían la compra y venta de acciones mediante algoritmos que operaban de manera simultánea en las bolsas de valores del mundo y tenían la capacidad de realizar múltiples transacciones con una velocidad impresionante. En adición, la postura económica dominante era (y es) que la regulación puede venir de la racionalidad del mercado, puesto que mediante innovaciones en formas complementarias de financiamiento se crean los mecanismos de equilibrio. Para esta formulación es clave la noción de que las diferentes formas de financiamiento para la canalización de bienes y servicios constituyen "productos" regulatorios y generan valor (Fox 2009, Smith 2011).

Dos ejemplos de "productos" que he caracterizado como "regulatorios" de acuerdo con el modelo económico basado en la oferta son los paquetes de hipotecas y los seguros en contra de deuda morosa (credit default swaps en inglés); ambos son considerados las acciones 'tóxicas" centrales de la crisis de 2008. Los paquetes de hipotecas fueron supuestamente combinaciones del crédito otorgado a consumidores de gran solvencia junto con el crédito aprobado para deudores de mediano a alto riesgo. El producto resultante fue una inversión en la que las hipotecas primarias de los deudores solventes operaban como garantías de una compensación adecuada para la compra del producto frente a las otras hipotecas de más riesgo incluidas en el paquete. También existían seguros para este tipo de inversión, donde los inversionistas podían comprar el seguro aun cuando no eran propietarios del bien asegurado (en este caso, del crédito otorgado); es decir, que existía la posibilidad de apostar a que la inversión iba a quebrar y recibir una compensación en caso de que el crédito no se pudiese pagar aun cuando el asegurado no era el deudor.

En 2008, estos dos "productos" no parecían haber operado como reguladores. Al contrario, los paquetes de hipotecas permitieron ofrecer más y más financiamiento a deudores no solventes y el seguro en contra de deudores morosos permitió a los inversionistas apostar en contra de los paquetes de hipotecas y a favor de una crisis económica. En otoño de 2008, ambos procesos se volvieron críti- 
cos: tantas hipotecas se volvieron morosas que los paquetes perdieron su valor $\mathrm{y}$, por lo tanto, los seguros del crédito de los paquetes fueron reclamados. Los gobiernos tuvieron que intervenir para evitar un colapso económico. Aun con este esfuerzo, la crisis resultante fue devastadora: los bancos de Islandia quebraron, la tasa de desempleo nacional en los Estados Unidos llegó a ser superior a 10\%, millones de personas perdieron sus casas y las industrias de construcción y venta de bienes raíces entraron en una profunda crisis (Harvey 2010, 1-39).

Según los economistas que apoyan el modelo de demanda, la crisis fue un ejemplo de los riesgos de un modelo económico que privilegia la oferta y, por lo tanto, la desregulación del sector financiero. En adición, argumentaron que la intervención del gobierno no era suficiente para lograr el estímulo adecuado para generar empleo y proteger el patrimonio de los sectores medios de la sociedad. No obstante, el modelo de oferta ha continuado su dominio en la economía política de Estados Unidos y países como el Reino Unido en Europa. Ahora, argumentan que las deudas en estos países son el problema central y la mejor manera de resolverlo es reducir el papel del gobierno en las áreas de seguro social, educación y salud; es decir, reducir el gasto del gobierno mediante la reducción de sus funciones y aumentar las condiciones de participación del sector privado mediante desregulación y la reducción de impuestos.

\section{DivUlgaCión DE CONOCIMIENTO Y LOS MODELOS DE OFERTA Y DEMANDA}

La crisis de la economía de 1929 condujo a una transición en la que se dejó una economía orientada a la oferta asociada con "los locos años veinte" y el entonces presidente de los Estados Unidos, Herbert Hoover, inició el desarrollo de un modelo keynesiano de la economía dentro de las economías nacionales. No obstante, en la década de los setenta del siglo pasado, los problemas para las economías nacionales crearon las condiciones para otra transición. Entre 1970 y 1990 , el dominio de los modelos de la economía política basada en la demanda cedió posición, de nuevo, a modelos orienta- 
dos por la oferta y particularmente por el control financiero de la oferta. En este mismo periodo (setenta-noventa) hubo, también, una revolución tecnológica. El resultado paradójico es que contamos en la actualidad con tecnologías de información y comunicación que posibilitan accesos más y más sofisticados e innovadores del producto acumulado del conocimiento humano pero, a la vez, la desregulación de los controles sobre los procesos para financiar la oferta de este conocimiento acumulado de la humanidad ha resultado en nuevas formas de exclusión social: a) el aumento en los costos de la educación mediante la privatización de la educación básica y superior; b) la evolución de monopolios y duopolios privados en países como México, tanto por la privatización de paraestatales, como por el fortalecimiento del dominio privado del mercado de los medios de radio y televisión por unas parentelas; y c) la creación de una población "excedente" cuya integración a una cultura nacional mediante programas públicos de salud, educación y acceso a créditos deja de ser parte de la política del Estado (Smith 2011).

Un ejemplo, entre muchos, es la transición de las revistas científicas mediante su integración en grandes empresas editoriales. Según la Asociación de Bibliotecas de Investigación en la Unión Americana, las suscripciones a revistas científicas y la compra de libros científicos entre 1986 y 2002 sufrieron los siguientes cambios: a) el número de revistas académicas en el mundo aumentó $58 \%$ y la tasa de publicación de monografías o libros se duplicó, pero el número de suscripciones a revistas académicas en bibliotecas de investigación de los Estados Unidos disminuyó 5\%; y b) las mismas bibliotecas gastaron $227 \%$ más en suscripciones a revistas en 2002 que en 1986, mientras el índice de precios para los consumidores de la Unión Americana sólo aumentó 62\% en el mismo periodo. ${ }^{1}$

Robert Darnton nos ofrece una visión aun más alarmante de este proceso: en los Estados Unidos el costo promedio de una suscripción a una revista académica en 1974 era de 54.86 dólares americanos y

${ }^{1}$ Citado en Appel y Servales 2004, "Creating Cultural Change in Scholarly Communication Systems”, Anthropology News, 12. 
en 2009 el promedio era 2,031 dólares por una revista norteamericana y 4,753 por una revista extranjera. Éste es un incremento diez veces mayor a la tasa de inflación en los Estados Unidos. Además Darnton reporta que tres años después del aumento de $227 \%$ en los veinte años entre 1986 y 2006, los precios de suscripciones habían aumentado otro 75\%, llegando a 302\%. Uno de los resultados de este control de la oferta de revistas académicas y el subsecuente crecimiento en los costos de suscripción institucional es que las bibliotecas de investigación han tenido que reducir sus adquisiciones de monografías. Hasta el año 2000, los departamentos de publicaciones universitarios de los Estados Unidos podían anticipar que las bibliotecas de investigación nacional comprarían 800 ejemplares de cada monografía editada, pero a partir del nuevo siglo este número se redujo a 300 o 400 ejemplares.

Darnton, también, presenta los costos asombrosos de revistas como Tetrahedron (química, 39,082 dólares anuales) y el Journal of Comparative Neurology (medicina, 27,465 dólares). Agrega que los márgenes de ganancia para las casas editoriales de revistas de ciencias, tecnología y medicina están entre 30 y $40 \%$ anuales, aun cuando dichas casas editoriales no agregan casi nada de valor a las revistas: las revistas académicas contienen los resultados de investigaciones financiadas con fondos públicos y tienen comités editoriales y dictaminadores que realizan sus trabajos especializados de manera gratuita. ${ }^{2}$

De nuevo, el modelo de la economía política basada en el control financiero de la oferta posibilita que las casas editoriales como Sage o Wiley o Elsevier puedan tomar control de la oferta de revistas creadas por académicos con material producido por investigaciones financiadas con fondos públicos. En adición, los académicos se encargan del proceso de organizar y realizar la revisión de pares de los trabajos producidos para publicación. Pero son las casas editoriales las que convierten todo este proceso de valor agregado en ganancias. Mediante el financiamiento de la canalización de las revistas, tanto a los suscriptores individuales, como a paquetes de ba-

\footnotetext{
${ }^{2}$ Robert Darnton, New York Review of Books, 23 de diciembre, 2010.
} 
ses de datos electrónicos ofertados a instituciones y, ahora, mediante sistemas sofisticados de búsqueda dentro de sus páginas de internet, supuestamente otorgan valor a las revistas, lo que justifica los altos precios de las subscripciones. Quizás el ejemplo más contemporáneo de este tipo de proceso, que hasta ahora no se ha logrado consumar en un negocio, es el proyecto de Google Book Search.

\section{LAS TIC Y EL PROYECTO DE GOOGLE}

Entre el 12 de junio de 2008 (apenas unos meses antes de la crisis económica arriba mencionada) y el 28 de abril de 2011, Robert Darnton escribió seis artículos en el New York Review of Books, así como respondió a nueve comentarios a sus escritos que giraron en torno al proyecto de Google de digitalizar el patrimonio mundial de libros publicados. En su primer trabajo ofreció una reflexión como historiador y como director de la Biblioteca en la Universidad de Harvard $^{3}$ acerca de lo que podemos clasificar como las tecnologías de información y comunicaciones para, luego, presentar el proyecto de Google Books y discutir posibles problemas con el proyecto.

La cronología de la evolución de las tecnologías de información y comunicaciones es impresionante. Una de las primeras TIC es la escritura (hace 6,100 años) que evolucionó en sistemas jeroglíficos (hace 5,300 ańos) y alfabéticos (3,000 ańos). Luego, hace unos 1,800 años, los lienzos y tabletas se complementaron con los códices con páginas y el desarrollo de las convenciones de la puntuación y la separación de palabras. En el año 1045 en China y en 1230 en Corea hubieron innovaciones en el uso de las letras de imprenta movibles (China, madera y Corea, metal), pero Darnton nota que la revolución de la imprenta inicia en Gutenberg en 1450 y se mantiene como la norma tecnológica durante casi cuatro siglos con notables cambios en el tamańo de la población de lectores. En la mitad del siglo XIX, se introdujo la impresión con máquinas de vapor y

${ }^{3}$ Especialista en la historia de Francia en el siglo xvıı así como en la historia del libro, Darnton es profesor emérito de Princeton y Carl H. Pforzheimer University Professor en Harvard. 
cambios dramáticos en la fabricación industrial de papel. Las innovaciones más recientes, sin embargo, giran en torno a la creación del internet entre 1974-1981, seguida por el desarrollo de los motores de búsqueda (Google en 1998) y los algoritmos para establecer las jerarquías de relevancia (Google en 2007) en la determinación del orden de las entradas (los hits) en cualquier tipo de búsqueda. A fines del siglo xx, el internet ya era la fuente más importante de consulta para información, rebasando libros, periódicos, radio y televisión.

Darnton nota que los intervalos entre estos desarrollos son impresionantes: de los inicios de la escritura a los códices, 4,300 ańos; de códice a letras de imprenta movibles, 1,150 años; de letras movibles a internet, 454 años; de internet a motores de búsqueda, 19 años; de motores de búsqueda a los algoritmos para establecer las jerarquías de relevancia, 7 años (Darnton 2008). En los cambios vertiginosos de los últimos años, Google ha sido uno de los protagonistas más importantes y la introducción de Darnton evalúa el proyecto de Google iniciado en 2004.

Desde 2004, Google iniciaba un programa ambicioso de digitalización de libros, provocando el litigio sobre derechos por parte de organizaciones de autores y editores en 2005. No obstante, en 2006, Google firmó un acuerdo con cinco bibliotecas de investigación (Stanford, Harvard, Chicago, Michigan y el Bodelian en Oxford) para digitalizar sus colecciones. En este mismo periodo existían otras iniciativas para digitalizar obras y crear bases de datos electrónicas: a) "Ilustración electrónica" -la digitalización de la correspondencia de Voltaire, Rousseau, Franklin y Jefferson con la eventual integración de la correspondencia de otros intelectuales en la base-; b) el proyecto de "Memoria americana" de la biblioteca del Congreso de los Estados Unidos; y c) el proyecto nombrado, "Valle de la Sombra" en la Universidad de Virginia. No obstante, el Google Book Search era el proyecto más grande y ambicioso que desde su inició provocó la intervención de autores y editores preocupados por sus derechos.

Darnton en este primer ensayo consideraba ocho áreas donde Google Book Search podría tener problemas, pero en general estaba a favor de la iniciativa y subrayó que no era un proyecto que tomaría 
el lugar de las bibliotecas de investigación, sino sólo facilitaría el acceso a los libros del mundo. Sus preocupaciones sobre el proyecto de Google son los siguientes:

1. El problema de la exclusión de textos importantes en el proceso de selección para el desarrollo de la colección.

2. La representatividad de la colección dado que, por ejemplo, en los Estados Unidos existen 543 millones de volúmenes en bibliotecas de investigación o, por ejemplo, en 2006 se publicaron 291,920 nuevos títulos en aquel país.

3. El problema de derechos de autor. La ley de 1978 y su extensión en 1998 protege la mayoría de los libros publicados después de 1923 y los derechos abarcan la vida del autor más setenta años. Dos organizaciones (una de autores y otra de editores) hicieron demandas de acción de clase en contra de Google en 2005 en relación con la violación de sus derechos mediante la digitalización y divulgación de partes de los libros todavía protegidos por las leyes de derechos.

4. Muchas de las bibliotecas de investigación han desarrollado sus colecciones durante siglos, mientras Google, como corporación, es una novedad emergente con una estabilidad todavía sin la prueba de los años.

5. El problema de errores en el proceso de digitalización que puede resultar en la omisión de páginas o de una imagen digital sin la definición requerida.

6. La preservación de la colección digital.

7. La selección de la edición de un libro más apropiada para su digitalización cuando el equipo de Google tiene técnicos sin formación en la bibliotecología.

8. La importancia de tener acceso a la composición material de textos; información que no puede ser captada mediante la digitalización electrónica.

En este primer ensayo, Darnton sólo estaba señalando la importancia de la continuidad de las bibliotecas de investigación y el hecho de que la iniciativa de Google, aun cuando importante, no iba a subs- 
tituir a estas bibliotecas y, de hecho, como proyecto tenía varias áreas de problemas todavía no resueltas. Pero en los cuatro ensayos publicados entre 2009 y 2010, Darnton ya estaba en contra del proyecto de Google Book Search y sumamente preocupado por una coyuntura.

Este cambio en la postura de Darnton resultaba de las dos demandas levantadas en contra de Google por un gremio de autores en septiembre y una asociación de editores en octubre de 2005. El 28 de octubre de 2008 las demandas fueron resueltas mediante un acuerdo entre Google y los demandantes. Según el acuerdo, habría la creación de un Registro de Derechos de Libros (Books Rights Registry) que representaría los intereses de los autores. La base de datos de Google ofrecería acceso a los libros en su mayoría con derechos reservados y fuera de imprenta mediante diferentes tipos de licencias: una licencia institucional para universidades; una licencia de acceso público para bibliotecas públicas; y una licencia de consumidor para individuos. La licencia de acceso público permitiría la instalación de una terminal de computadora en cada biblioteca pública con acceso gratis a los materiales. En contraste, las otras licencias tendrían un costo y $63 \%$ de las ganancias de las licencias iría a los autores, mientras $37 \%$ se quedaría para Google. El acceso a los libros de dominio público sería gratuito.

Este acuerdo tenía que ser aprobado por la Corte Federal del Distrito del Sur de Nueva York y Darnton en el primero de sus tres artículos críticos sobre el proyecto de Google estaba abogando por la no aprobación del acuerdo (un cuarto artículo en este periodo aboga por la creación de bibliotecas digitales públicas nacionales). Su mayor preocupación tuvo que ver con el hecho de que el acuerdo entre Google y los autores se diera a raíz de una demanda de acción de clase; es decir que si la Corte lo aprobaba aplicaría a todos los autores. En tal caso, Google no tendría que responder a ninguna demanda de personas con derechos de autor, mientras cualquier otra empresa de divulgación mediante digitalización podría ser demandada. En adición, la escala de digitalización de libros por parte de Google era exponencialmente mayor que cualquier otro esfuerzo público, como, por ejemplo, el Open Knowledge Commons. En fin, Google tendría un control monopólico sobre el acceso a libros digitales. 


\section{LAS TIC Y LA DIVULGACIÓN}

En casi todos sus ensayos para el New York Review sobre el proyecto de Google, Robert Darnton observa que su argumento suele moverse entre lamentaciones y entusiasmo utópico. Por un lado, la evolución de las TIC permite un acceso sin precedentes al conocimiento acumulado de la humanidad. Por lo tanto, no es sorprendente que tres distinguidos estudiosos de la Universidad de Michigan, Paul N. Courant, decano de bibliotecas, Harold T. Shapiro, profesor de política pública y Arthur F. Thurnau, profesor de economía y de información, respondieran al segundo ensayo donde Darnton argumentó en contra del proyecto de Google y el acuerdo realizado entre Google y los autores. Subrayaron en su respuesta al ensayo de Darnton que las acciones de Google no deberían interpretarse como parte de una práctica monopólica sino, al contrario, representan el inicio de un proceso de instituir una república de letras en beneficio de toda la humanidad.

Por otro lado, Google tenía un creciente control sobre el acceso y los términos de acceso a los libros digitalizados constitutivos de esta ilusión de una república de letras. Y, de nuevo, no es sorpresivo que en respuesta al primer ensayo de Darnton en el que apoyó la iniciativa de Google, Jean-Claude Guédon, profesor de literatura comparativa de la Universidad de Montreal, replicase insistiendo en el hecho de que la copia digital universal de los libros de Google no permitía su transformación a un formato compatible con programas y aplicaciones distintos de los programas y aplicaciones de Google, de manera que, efectivamente, monopolizaba la vía de acceso a los libros que digitaliza.

En adición, la respuesta al proyecto de Google desde Europa era aún más crítica. Apenas un mes después del anuncio del 14 de diciembre de 2004 que Google digitilizaría 15 millones de libros de cinco bibliotecas de investigación de la Unión Americana y el Reino Unido, el entonces director de la Biblioteca Nacional de Francia, Jean-Noël Jeanneny, publicó una crítica en las páginas de Le Monde, ${ }^{4}$ un texto que unos meses después sería ampliado en un li-

${ }^{4} 22$ de enero 2005, "Quand Google defie l'Europe" < http://www.lemonde.fr/cgi- 
bro que capta el proyecto de Google y sus implicaciones desde el punto de vista de la política cultural de Europa y especialmente de Francia. ${ }^{5}$ El enfoque central de la crítica de Jeanneny es el sesgo cultural y lingüístico del proyecto Google, tanto en la selección de textos que favorecen el inglés, como en los algoritmos de búsqueda que diseña Google; ambos actos cómplices con la hegemonía anglófona en publicaciones científicas y humanísticas. Notó, por ejemplo, que una búsqueda en Google Books de Víctor Hugo, Dante, Cervantes y Goethe produjo sólo traducciones de sus obras en inglés (Jeanneny 2006, 11, citado en Bearman 2006). En adición, Jeanneny exploró, tanto el proceso de búsqueda propuesta por Google en términos de sus poderes para una descontextualización de las obras en demérito de sus contenidos, como las consecuencias de poner un proyecto cultural tan importante como una biblioteca digital universal bajo el control de una corporación privada. Como veremos, los argumentos de Jeanneny aparecieron al centro de un memorando entregado por Francia a la corte federal de los Estados Unidos en contra del proyecto de Google.

Los ensayos de Darnton documentan, tanto el entusiasmo utópico, como las lamentaciones acerca de la empresa de Google Book Search y sus implicaciones. Constituyen una documentación valiosa para reflexionar sobre el futuro de proyectos públicos de bibliotecas virtuales que pudieran abrir el acceso a los libros del mundo. Tales proyectos ya son factibles. El 28 de marzo de 2011, la Corte del Distrito Sur de Nueva York falló en contra del acuerdo entre Google y las dos asociaciones de autores y de editores sobre sus demandas de acción de clase. La decisión de la Corte respondió a la creciente oposición a la propuesta de Google que mediante memorando y observaciones señalaron, tanto los problemas específicos del proyecto de Google, como los más generales para cualquier proyecto de digitalización de los libros del mundo. Los gobiernos de Francia y

bin/ACHATS/acheter.cgi?offre=ARCHIVES\&type_item=ART_ARCH_30J\&objet_ id $=885549>$.

${ }^{5}$ Quand Google défie l'Europe: Plaidoyer pour un sursaut (abril 2005), traducido al inglés con el titulo, Google and the Myth of Universal Knowledge: A View from Europe (Teresa Lavendar Fagan trad.) Universidad de Chicago 2006. 
Alemania solicitaron que la Corte rechazara el acuerdo o, al menos, reconociera que dicho acuerdo no aplica a sus respectivos ciudadanos. Darnton nota que el documento de Francia criticó "el poder concentrado y no regulado" del acuerdo, mientras que Alemania criticó "un acuerdo de orientación comercial negociado de manera oculta por tres partes interesadas, el Gremio de Autores, la Asociación de Editores Americanos y Google Inc.". Darnton enumera las objeciones de ambos gobiernos:

1. El acuerdo otorga a Google control monopólico sobre libros huérfanos (libros que no son de dominio público pero están fuera de imprenta y las direcciones y el estatus de sus autores no son conocidos) aun cuando no tiene ningún derecho sobre estos libros.

2. Los derechos inherentes de los autores no son respetados en el acuerdo puesto que son ellos los que deben notificar a Google para indicar que no aceptan el acuerdo: si no lo hacen, serán tratados como aceptación de acuerdo con los términos del Registro de Derechos de Libros (Books Rights Registry).

3. El acuerdo contiene una cláusula para asegurar que en cualquier uso comercial de los libros digitalizados, Google siempre tendrá las mejores condiciones frente a otros competidores.

4. Google mantiene el derecho de censura de su base de datos mediante la exclusión de hasta $15 \%$ de las obras digitalizadas.

5. Mediante el uso de los algoritmos de Google para establecer los precios, los intereses comerciales de Google predominan sobre el bien del público.

6. Las relaciones entre Google y los representantes de los autores y editores en el Books Rights Registry son reservadas: el público no puede asistir a sus reuniones.

La Federación Internacional de Asociaciones de Bibliotecas (IFLA), el Buró Europeo de Biblioteca, Información y Documentación Asociados (EBLIDA), y la Ligue des Bibliothèques Européennes de Recherche (LIBER) ante La Comisión Europea presentaron los mismos puntos en contra del acuerdo, pero haciendo hincapié en el riesgo de que "una proporción grande del legajo mundial de libros en el formato digital estarán bajo el control de una sola entidad corporativa". 
El Departamento del Estado de los Estados Unidos criticó el acuerdo en términos de la ventaja injusta de Google frente a otros competidores así como su potencial control de facto sobre los libros huérfanos: El acuerdo eliminaría los riesgos de demandas a Google sobre derechos de autor, pero no para otras empresas. No obstante, el Departamento también reconoció el beneficio potencial del proyecto de Google y, por lo tanto, en vez de rechazar el acuerdo recomendó cambios:

1. Requerir que autores con derechos vigentes soliciten su participación en el acuerdo y no al revés.

2. No distribuir las ganancias de la venta de libros huérfanos a las partes del acuerdo sino usar el dinero para buscar a los autores con derechos durante un tiempo significativo.

3. Designar guardianes para proteger los intereses de los autores de libros huérfanos.

4. Procurar que los competidores potenciales de Google puedan tener acceso a las obras huérfanas sin problemas de demandas de violación de derechos.

5. No permitir a Google usar libros fuera de imprenta en nuevos productos comerciales sin permiso de los autores o las personas con derechos de autor.

Fue en el contexto de esta oposición, especialmente del memorando del Departamento del Estado, que Google y las asociaciones de autores y editores demandantes solicitaron una prórroga del juicio para reelaborar su acuerdo. El 13 de noviembre de 2009 registraron un acuerdo revisado con cambios en respuesta a las observaciones críticas de varios memorandos. Según Darnton, los cambios en la nueva versión del acuerdo indicaban que las recomendaciones del Departamento de Estado les sirvieron para su elaboración. Propusieron guardianes nombrados por la Corte para representar a los autores de libros huérfanos. De igual manera, acordaron que las ganancias de la venta de libros huérfanos podrían ser utilizadas durante diez años para la búsqueda de las personas que tienen los derechos de autor y, luego, podrían ser distribuidas para obras de caridad determinadas por la Corte. A la vez, aceptaron que los nuevos productos comerciales basados en libros fuera de impren- 
ta podrían ser desarrollados por competidores de Google, pero sólo para consumidores individuales. Google tendría control exclusivo sobre productos para suscripciones institucionales. Google, también, tendría el derecho de considerar autores o personas con los derechos de autores como partícipes en el acuerdo si formalmente no habían comunicado su desacuerdo de participación.

El 18 de febrero de 2010, el juez Chin de la Corte Federal del Distrito Sur de Nueva York convocó un foro para recibir las opiniones y observaciones sobre el nuevo acuerdo (Amended Settlement Agreement, ASA). Casi trece meses después, el 23 de marzo de 2011, el juez Chin rechazó este acuerdo. Como notó Darnton, todavía no se sabe si Google podrá rescatar su proyecto mediante otros mecanismos, tanto jurídicos (un nuevo acuerdo con autores y editores), como de innovación con las Tic (nuevos algoritmos para búsquedas y organización de texto). No obstante, Darnton argumenta que ahora la iniciativa para la divulgación del conocimiento acumulado en el legajo de libros mediante las nuevas tecnologías de información y comunicaciones debe ser pública y dirigida a la creación de bibliotecas digitales nacionales públicas.

\section{Bibliotecas Digitales Nacionales Públicas}

Darnton nota que ya existen varias iniciativas nacionales para la creación de una biblioteca digital pública. La Biblioteca Nacional de los Países Bajos cuenta con un programa para la digitalización en los próximos diez ańos de todos los textos en holandés (libro, periódico, revista) creados desde 1470 al presente. Noruega tiene un proyecto paralelo y Finlandia, Australia y Japón están digitalizando la totalidad de sus colecciones en las bibliotecas nacionales. En Francia, el presidente Sarkozy autorizó una bolsa de 750 millones de euros para la digitalización del patrimonio cultural francés. En adición, existen proyectos de consorcios de universidades dedicados en gran medida a la preservación de sus colecciones mediante la digitalización.

El obstáculo más grande en todas estas iniciativas, según Darnton, no es fiscal sino jurídico. En el caso de los Estados Unidos y de acuerdo con la ley de derechos existe una cantidad considerable de 
libros fuera de imprenta, pero todavía cubierta por derechos de autor. De los libros publicados entre 1923 y 1964, por ejemplo, la mayoría son libros huérfanos: están fuera de imprenta, no se conoce la dirección o el estatus del autor, pero los derechos todavía pertenecen al autor o a sus descendientes. Para incluir estos libros en una biblioteca digital pública se necesitaría una legislación específica mediante la intervención del Congreso. Éste es el caso en la mayoría de los países donde el objetivo es la creación de una biblioteca digital pública nacional con los libros de dominio público y los libros huérfanos, dejando en circulación comercial los libros con derechos de autor vigentes y todavía en procesos de edición. No obstante, en países como Noruega y los Países Bajos donde la escala de publicación en noruego y holandés lo permiten, se contempla también incluir mediante compensación las publicaciones con derechos vigentes en la colección digital.

Darnton observa que la creación de las bibliotecas digitales públicas nacionales en Europa creó la posibilidad de otro programa denominado: "Europeana". Este programa opera en tres niveles: primero, bibliotecas particulares digitalizan sus colecciones; segundo, centros nacionales o regionales integran estas colecciones en una base de datos central; y, tercera, Europeana crearía una red de los 27 países participantes. Así, el usuario buscaría el libro dentro de la red y llegaría a la versión digital en un sitio dentro de la red para descargarla en su computadora. Darnton sugiere que un sistema semejante podría funcionar para la Unión Americana y nota que la donación de los dos millones de libros de dominio público ya digitalizados por Google podría constituir la aportación inicial de Google como participante en un nuevo proyecto para la creación de una biblioteca digital pública nacional.

\section{CONCLUSIÓN: DEMANDA Y OFERTA}

El dominio del capital financiero en la canalización de la oferta de los bienes y servicios es característico de una economía global que ha emergido mediante la desregulación de las economías nacionales. Las innovaciones en las tecnologías de información y comunicacio- 
nes constituyen una de las condiciones de la posibilidad para el desarrollo de un mercado global en el que nuevos productos giren en torno a diferentes tipos de financiamiento de la oferta (paquetes de hipotecas, por ejemplo) y sus derivados (seguros del crédito moroso para los paquetes o credit default swaps en inglés). La velocidad del movimiento global de crédito, los algoritmos de compra y venta que operan en nanosegundos en operaciones entre Nueva York, Chicago, Londres y las demás bolsas de valores en el mundo; todas requieren las nuevas TIC para instrumentarse. Paradójicamente, estas mismas TIC son, también, las condiciones de posibilidad para abrir el acceso al conocimiento acumulado contenido en los textos producidos desde hace cinco milenios.

Como hemos visto con las revistas académicas y con el proyecto de Google Book Search, las dos tendencias (control financiero de oferta e institucionalización de garantías de acceso a información) se encuentran en un conflicto que evoca la visión hegeliana de la dialéctica histórica. El entusiasmo utópico por un nuevo acceso abierto al conocimiento se convierte en un lamento acerca de un nuevo control comercial sobre el derecho a la información. No obstante, existen nuevas iniciativas que dan lugar a cierto optimismo. Por ejemplo, en los Estados Unidos varias redes de científicos han creado revistas académicas en que, tanto el control de la producción, como el control de la distribución y venta quedan en manos de los científicos. Así, es posible no sólo reducir los costos de las suscripciones sino ofrecer acceso digital abierto. Hace dos años se creó una coalición para publicaciones de acceso abierto entre varias universidades. La coalición opera para dirigir la producción de revistas académicas e inclusive ofrecer subsidios a autores sin el financiamiento de universidades o fundaciones para sus investigaciones. ${ }^{6}$

Algo semejante pasa en México donde la Red de Revistas Científicas de América Latina, el Caribe, España y Portugal ofrece acceso abierto a 758 revistas académicas. ${ }^{7}$ De manera semejante, hay un número importante y creciente de iniciativas para la creación de

${ }^{6}$ The Compact for Open-Access Publishing Equity (cOPE).

${ }^{7}$ http://redalyc.uaemex.mx/ 
colecciones digitales de textos para alimentar bibliotecas públicas y bibliotecas de investigación con acceso abierto. Algunos de los programas públicos en Europa y Los Estados Unidos ya se han descrito arriba y en México acciones paralelas están en proceso en archivos y bibliotecas de investigación. En adición, existen redes de instituciones con sus consejos como el Consejo Asesor de Recursos de Información (CARI) de los 27 Centros Públicos de Investigación Conacyt que operan para coordinar mejor el acceso a las publicaciones académicas y reducir el costo de este acceso para cada institución.

La paradoja existe, no obstante, entre el dominio actual del modelo económico orientado a la oferta y sus condiciones en países como México, los Estados Unidos o el Reino Unido, por un lado, y, por otro, las posibilidades de acceso rápido al conocimiento acumulado de la humanidad registrado en textos mediante búsquedas selectivas por palabras claves, autores, conceptos, patrones anteriores de selección, afinidades entre temas, jerarquías de relevancia, y muchos criterios más. Si, como David Harvey (1989) ha argumentado, estamos entrando en una época en la cual el dinero es la única medida de valor, entonces es probable que la tendencia de privilegiar el control financiero de la oferta provocará que más empresas busquen controlar el acceso al producto acumulado del conocimiento de las ciencias y humanidades. No obstante, todavía existen posibilidades de organización dentro de la esfera pública para asegurar que las TIC nos permitan fortalecer el acceso abierto a los resultados de milenios de creatividad humana registrados en textos.

\section{REFERENCIAS CITADAS}

Appel y Servales, "Creating Cultural Change in Scholarly Communication Systems", Anthropology News, 12, 2004.

Bearman, David, "Jean-Noël Jeanneney's Critique of Google: Private Sector Book Digitization and Digital Library Policy”, D-Lib Magazine, diciembre, vol. 12, núm. 12, 2006.

DARnTON, Robert, "The library in the new age", New York Review of Books, 12 de junio, 2008. , "Google \& the future of books", New York Review of Books, 
12 de febrero, 2009.

"Google and the new digital future", New York Review of

Books, 17 de diciembre, 2009.

, "Can we create a National Digital Library?", New York Review of Books, 28 de octubre, 2010.

"The library: three jeremiads", New York Review of Books,

23 de diciembre, 2010.

, "Google's loss: the public's gain", New York Review of Books, 28 de abril, 2011.

Fox, Justin, The Myth of the Rational Market, Harper, Nueva York, 2009.

Harvey, David, The condition of Posmodernity, Cambridge, Blackwell, 1989.

, The enigma of capital and the crisis of capitalism, Nueva York, Universidad de Oxford, 2010.

Jeanneney, Jean-Noël, “Quand Google défie l'Europe”, Le Monde, 22 de enero de 2005.

, Google and the Myth of Universal Knowledge: A view from Europe, traducción de Teresa Lavendar Fagan, Chicago, Universidad de Chicago, 2006, 92 p.

Smith, Gavin, "Selective hegemony and beyond, populations with 'no productive function': a framework for enquiry", Identities. Global Studies in Culture and Power, vol. 18, núm. 1, 2011.

Intercambios con Robert Darnton:

GideAn, Jean-Claude, "Who will digitize the world's books?", New York Review of Books, 14 de agosto, 2008.

Courant, Paul N., Harold T. Shapiro, Arthur F. Thurnau, "Google and Books and Exchange", New York Review of Books, 26 de marzo, 2009. 